\title{
Long-term efficacy of vasodilating $\beta$-blocker in patients with acute myocardial infarction: nationwide multicenter prospective registry
}

Jaehoon Chung ${ }^{1}$, Jung-Kyu Han², Han-Mo Yang ${ }^{2}$, Kyung-Woo Park², Hyun-Jae Kang², Bon-Kwon Koo², Myung Ho Jeong ${ }^{3}$, Hyo-Soo Kim² ${ }^{2}$, and on behalf of investigators for Korea Acute Myocardial Infarction Registry (KAMIR)

${ }^{1}$ Division of Cardiology, Department of Internal Medicine, National Medical Center, Seoul; ${ }^{2}$ Cardiovascular Center, Department of Internal Medicine, Seoul National University Hospital, Seoul; ${ }^{3}$ Department of Internal Medicine, Chonnam National University Hospital, Gwangju, Korea

Received: April 5, 2020 Revised : May 6, 2020 Accepted: May 17, 2020

\section{Correspondence to} Jung-Kyu Han, M.D. Cardiovascular Center, Department of Internal Medicine, Seoul National University Hospital, 101 Daehak-ro, Jongno-gu, Seoul 03080, Korea

Tel: $+82-2-2072-4870$

Fax: $+82-2-766-8904$

E-mail: hpcrates@gmail.com https://orcid.org/0000-00020016-0747
Background/Aims: Long-term benefit of vasodilating $\beta$-blockers is unknown. This study aimed to investigate the long-term benefit of vasodilating $\beta$-blockers over conventional $\beta$-blockers in patients with acute myocardial infarction (AMI). Methods: Using nationwide prospective multicenter Korean Acute Myocardial Infarction Registry data, we analyzed 3-year clinical outcomes of 7,269 patients with AMI who received percutaneous coronary intervention (PCI) and $\beta$-blocker therapy. Patients were classified according to treatment strategy (vasodilating $\beta$-blockers vs. conventional $\beta$-blockers). The primary endpoint was a composite of cardiac death, myocardial infarction (MI), and hospitalization for heart failure (HF) at 3 years. Secondary outcomes were each component of the primary outcome. Propensity score matching was performed to adjust for differences of baseline characteristics.

Results: In 3,079 pairs (6,158 patients) of propensity score-matched patients, the primary outcome occurred significantly less in the vasodilating $\beta$-blockers group compared with the conventional $\beta$-blockers group (7.6\% vs. 9.8\%, $p=0.003$ ). Among the secondary outcomes, cardiac death occurred significantly less in the vasodilating $\beta$-blockers group than in the conventional group $(3.5 \%$ vs. $4.8 \%, p=$ $0.015)$. The incidence rates of MI (2.4\% vs. $3.0 \%, p=0.160)$ or hospitalization for HF (2.6\% vs. $3.2 \%, p=0.192$ ) were not significantly different between the two groups.

Conclusions: Vasodilating $\beta$-blocker therapy was associated with better clinical outcomes compared with conventional $\beta$-blocker therapy in AMI patients undergoing PCI during 3 years follow-up. Vasodilating $\beta$-blockers could be recommended preferentially for these patients.

Keywords: Beta-blocker; Myocardial infarction; Coronary artery disease; Percutaneous coronary intervention

\section{INTRODUCTION}

The use of $\beta$-blockers has been considered a fundamental treatment strategy for patients with acute myocardial infarction (AMI) [1-4]. In particular, newer generation $\beta$-blockers with vasodilating and antioxidant properties, such as carvedilol and nebivolol, possess theoretical benefits over conventional $\beta$-blockers. In a previous study conducted in diabetic patients with hypertension, carvedilol demonstrated favorable effects on glucose 
and lipid metabolism and reduced lipid peroxidation compared with atenolol [5]. In the Glycemic Effects in Diabetes Mellitus: Carvedilol-Metoprolol Comparison in Hypertensives (GEMINI) trial, carvedilol did not worsen glycemic control and improved insulin sensitivity, in contrast to metoprolol, in patients with type 2 diabetes mellitus and hypertension [6]. Unlike atenolol, nebivolol did not worsen insulin sensitivity in hypertensive patients with impaired glucose tolerance [7]. In addition to these distinctive metabolic features, both carvedilol and nebivolol improved endothelial function in contrast to conventional $\beta$-blockers $[8,9]$. Moreover, vasodilating $\beta$-blockers do not elevate central blood pressure by reducing vascular resistance [10-12]. In accordance with these advantages of vasodilating $\beta$-blockers, we previously reported for the first time the benefit of vasodilating $\beta$-blockers over conventional $\beta$-blockers in AMI patients undergoing percutaneous coronary intervention (PCI) for 1 year in terms of cardiac death and other clinical composite outcomes [13]. On the basis of the above-mentioned mechanistic background supporting the benefits of vasodilating $\beta$-blockers over conventional $\beta$-blockers, we can surmise that longer-term benefits of vasodilating $\beta$-blocker therapy would be greater. Herein we analyzed a nationwide prospective multicenter Korean Acute Myocardial Infarction Registry (KAMIR) to assess 3-year clinical outcome of $\beta$-blocker therapy according to its subtype.

\section{METHODS}

\section{Study population and study design}

This study was based on data from KAMIR, which is a nationwide multicenter prospective registry of patients with AMI in Korea [14]. The data were collected from each participating hospital and entered into the KAMIR database via a web-based electronic system. From November 2011 to November 2015, 13,104 consecutive patients with AMI were enrolled in KAMIR. Among the patients, those who died in the hospital $(n=503)$; who were not prescribed any $\beta$-blocker at discharge $(\mathrm{n}=$ $2,058)$; who were prescribed $\beta$-blockers other than carvedilol and nebivolol (vasodilating) as well as bisoprolol and metoprolol (conventional) at discharge $(\mathrm{n}=85)$; who did not undergo PCI but had undergone coronary artery bypass graft, thrombolysis, or medical therapy alone $(\mathrm{n}=$ 1,376); who did not revisit the hospital after discharge $(\mathrm{n}=$ 95); and whose prescribed $\beta$-blocker was either changed to a different class of $\beta$-blockers (from vasodilating to conventional, or vice versa) or was discontinued during the 3-year follow-up $(n=1,718)$ were excluded. As a result, 5,835 patients were excluded and 7,269 patients were analyzed in this study. The participants were divided into two groups according to the type of prescribed $\beta$-blocker: vasodilating $\beta$-blocker group (carvedilol, $\mathrm{n}=3,251$; nebivolol, $\mathrm{n}=357$ ) and conventional $\beta$-blocker group (bisoprolol, $\mathrm{n}=3,533$; metoprolol, $\mathrm{n}=128$ ). As this study was performed on the basis of registry data, propensity score matching was conducted to adjust for differences in baseline characteristics; finally, 3,079 pairs of propensity score-matched patients were created (Fig. 1). Our study was conducted according to the Declaration of Helsinki. The study protocol was approved by the Institutional Review Board of all centers (Seoul National University Hospital; SNUH IRB No. 1603-041-747), and written informed consent was obtained from all patients.

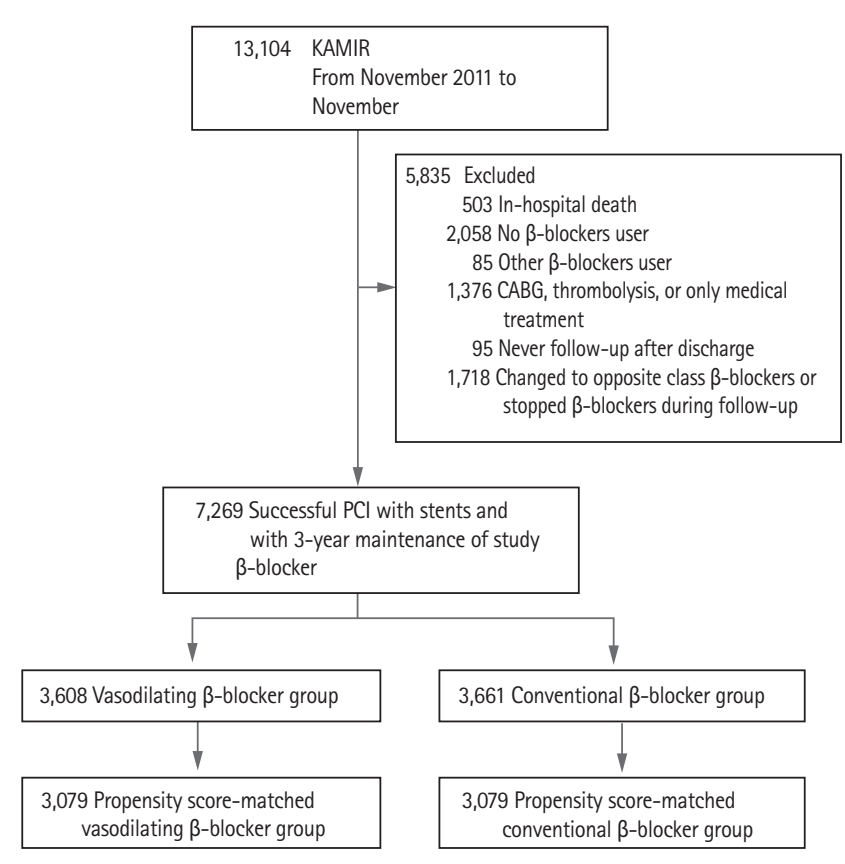

Figure 1. Flow chart of the group distribution for analysis. The propensity score was estimated with all variables shown in Table 1. KAMIR, Korean Acute Myocardial Infarction Registry; CABG, coronary artery bypass graft; PCI, percutaneous coronary intervention. 


\section{Clinical data collection}

Previous history of cardiovascular risk factors, medication at discharge were obtained. Diabetes mellitus was defined as previously diagnosed untreated diabetes mellitus or current treatment with oral hypoglycemic agents or insulin. Hypertension was defined as previously diagnosed untreated hypertension or current treatment with antihypertensive medications. Dyslipidemia was defined as previously diagnosed untreated dyslipidemia or current treatment with lipid-lowering agents. Chronic kidney disease was defined as estimated glomerular filtration rate less than $60 \mathrm{~mL} / \mathrm{min} / 1.73 \mathrm{~m}^{2}$ at the time of admission.

\section{Percutaneous coronary intervention procedure}

PCI was performed according to the current procedural guideline. All patients received $300 \mathrm{mg}$ loading dose of aspirin and $600 \mathrm{mg}$ loading dose of clopidogrel, 60 mg loading dose of prasugrel or $180 \mathrm{mg}$ loading dose of ticagrelor before or during PCI, unless they had previously received these antiplatelet drugs. During PCI, weight-adjusted unfractionated heparin was administered to patients to keep the activated clotting time between 250 and 350 seconds. There was no limitation on the selection of stent manufacturers, number of stents, use of glycoprotein IIb/IIIa receptor inhibitors, use of intravascular imaging device or post-dilatation after stent implantation. Pre- and post-PCI coronary flows were graded based on the thrombolysis in myocardial infarction (TIMI) grading system. Coronary lesions were classified according to the American College of Cardiology/American Heart Association coronary lesion classification system.

\section{Definitions and outcomes}

The primary outcome was a composite of cardiac death, recurrent non-fatal myocardial infarction (MI), or hospitalization for heart failure (HF) at 3 years. Secondary outcomes were each component of the primary outcome. All deaths were considered to be associated with a cardiac cause, unless a definite non-cardiac cause could be established. Recurrent MI was defined as recurrent symptoms of myocardial ischemia with new ischemic electrocardiographic changes compatible with MI or elevation of cardiac troponin values with at least one value above the 99th percentile upper reference limit. Hospi- talization for HF was defined as re-hospitalization as a result of worsening HF requiring more intensive care than continuation of usual treatment in the outpatient department. Clinical follow-up was routinely performed $6,12,24$, and 36 months from the index hospitalization by visiting hospitals and whenever any clinical event took place. The patient's physician identified all clinical events and the principal investigator of each hospital confirmed them.

\section{Statistical analysis}

The results were expressed as mean \pm standard deviation (SD) for continuous variables and as percentage for categorical variables. For continuous variables, Student's $t$ test was used to test the differences between the two treatment groups; for categorical variables, Pearson's chi-square test was used to compare the distribution between the two treatment groups. Because the $\beta$-blocker strategy was not randomized, a propensity score was used to adjust for potential selection or predisposition bias. The propensity score was estimated using multiple logistic regression analysis, with all variables shown in Supplementary Table 1. On the basis of the propensity score, the patients were selected by 1:1 matching without replacement using a greedy algorithm and the nearest available pair matching methods. A caliper width of 0.1 $\mathrm{SD}$ of the logit of the estimated propensity score was used for matching. The covariate balance achieved by propensity score-matching was assessed by calculating the absolute standardized differences in covariates between the two groups. After propensity score matching, the study end-points were demonstrated using the Kaplan-Meier survival curves and compared using the log-rank test. The Cox proportional hazards model was used to estimate the hazard ratio (HR) and 95\% confidence intervals (CI) for between-group comparison. To determine independent associations between the type of $\beta$-blocker and clinical outcomes, a multivariable Cox regression analysis was performed. In the multivariable Cox regression analysis, age, sex, hypertension, diabetes mellitus, dyslipidemia, smoking status (current or ex-smoker), chronic kidney disease, left main or left anterior descending artery as infarct-related artery, multivessel disease, left ventricular ejection fraction (LVEF), ST segment elevation MI (STEMI) or non-ST segment elevation MI (NSTEMI), and use of renin-angiotensin 
system blocker and statin were included as covariates, which were significant on univariable analysis and were generally considered clinically relevant. All analyses were two-tailed, and clinical significance was defined as $p<0.05$. All statistical analyses were performed using the statistical package SPSS version 22.0 (IBM Co., Armonk, $\mathrm{NY}, \mathrm{USA}$ ), and R programming language version 3.13 ( $\mathrm{R}$ Foundation for Statistical Computing, Vienna, Austria).

\section{RESULTS}

\section{Baseline characteristics}

Whole population

Of the 7,269 study patients, 3,608 (49.6\%) were treated with vasodilating $\beta$-blockers such as carvedilol and nebivolol, while the other 3,661 (50.4\%) were treated with conventional $\beta$-blockers such as bisoprolol and metoprolol. The mean age of all patients was $62.8 \pm 12.4$ years. Of the total patients, 5,519 (75.9\%) were men, 1,544 (21.2\%) had diabetes mellitus, and 3,863 (53.1\%) were STEMI. Renin-angiotensin system blocker was prescribed to $6,305(86.7 \%)$ patients, and statin was prescribed to 6,962 (95.8\%) patients. Intracoronary imaging device was used in 1,549 (21.3\%) patients. A total of 3,731 (51.3\%) patients had multivessel coronary artery disease. The specific baseline clinical and procedural characteristics are shown in Supplementary Table 1.

\section{Propensity score-matched population}

Propensity score matching was performed to adjust for differences of baseline characteristics. A total of 3,079 pairs of propensity score-matched patients were analyzed in this study. The C-statistic for the propensity score model was 0.519 , demonstrating that selection of $\beta$-blocker types was relatively random. The $p$ value of Hosmer-Lemeshow goodness of fit for the propensity score model was 0.210 . There were no significant differences in the baseline clinical and procedural characteristics between the two groups, and the absolute standardized differences were $<10.0 \%$ in all covariates in the propensity-matched patients. The baseline clinical, angiographic, and procedural characteristics of the study patients are provided in Table 1 . The mean age of the study patients was $62.6 \pm 12.4$ years. Of the total patients, 4,702 (76.4\%) were men, 1,263 (20.5\%) had diabetes mellitus, and 3,306 (53.7\%) were STEMI.

\section{Clinical outcomes}

Whole population

The median follow-up duration was 1,097 days (interquartile range, 1,049 to 1,129). At 3 years, 641 primary events (8.8\%) defined as a composite of cardiac death, MI, or hospitalization for HF occurred. In the Kaplan-Meier curve analysis, primary events occurred significantly less frequently in vasodilating $\beta$-blocker group than in the conventional $\beta$-blocker group (7.8\% vs. $9.8 \%$, vasodilating vs. conventional, respectively; log rank $p=$ 0.003). The difference in primary outcome between the two groups was mainly driven by cardiac death (3.5\% vs. $4.8 \%, \log \operatorname{rank} p=0.008)$. The incidence rates of $\mathrm{MI}(2.5 \%$ vs. $3.1 \%, \log \operatorname{rank} p=0.140$ ) and hospitalization for $\mathrm{HF}$ (2.8\% vs. $3.1 \%, \log$ rank $p=0.336)$ were not significantly different between the two groups (Supplementary Table 2 and Supplementary Fig. 1). In the multivariable Cox regression analysis, use of vasodilating $\beta$-blocker was an independent predictor of the primary outcome after 3 years in AMI patients who underwent PCI (adjusted HR, o.80; 95\% CI, 0.68 to $0.94 ; p=0.006$ ) (Supplementary Table 2). The other independent predictors were age, diabetes mellitus, hypertension, dyslipidemia, chronic kidney disease, multi-vessel disease, and LVEF.

Propensity score-matched population

The median follow-up duration was 1,097 days (interquartile range, 1,050 to 1,130). After 3 years, the incidence of primary outcome was significantly lower in the vasodilating $\beta$-blocker group (7.6\% vs. $9.8 \%, \log \operatorname{rank} p=$ $0.003)$. The difference was also mainly driven by cardiac death (3.5\% vs. $4.8 \%, \log \operatorname{rank} p=0.015)$. The incidence rates of MI (2.4\% vs. 3.0\%, log rank $p=0.160)$ and hospitalization for $\mathrm{HF}(2.6 \%$ vs. $3.2 \%, \log$ rank $p=0.192)$ were not different between the two groups (Table 2 and Fig. 2). On multivariable Cox regression analysis, the use of vasodilating $\beta$-blockers was found to be an independent predictor of primary outcome after 3 years (adjusted HR, 0.76; 95\% CI, 0.64 to $0.90 ; p=0.002$ ) (Table 2). The other independent predictors were age, hypertension, chronic kidney disease, left main or left anterior descending artery as infarct related artery, multivessel disease, and LVEF. 
Table 1. Baseline characteristics of propensity score-matched patients according to treatment at discharge and during follow-up

\begin{tabular}{|c|c|c|c|c|}
\hline Variable & $\begin{array}{l}\text { Vasodilating } \beta \text {-blockers } \\
\qquad(\mathrm{n}=3,079)\end{array}$ & $\begin{array}{c}\text { Conventional } \\
\beta \text {-blockers }(n=3,079)\end{array}$ & $p$ value & $\begin{array}{c}\text { Standardized } \\
\text { difference }\end{array}$ \\
\hline \multicolumn{5}{|l|}{ Demographics } \\
\hline Age, yr & $62.6 \pm 12.3$ & $62.7 \pm 12.4$ & 0.618 & 1.3 \\
\hline Male sex & $2,352(76.4)$ & $2,350(76.3)$ & 0.952 & 0.2 \\
\hline \multicolumn{5}{|l|}{ Coronary risk factors } \\
\hline Diabetes mellitus & $620(20.1)$ & $643(20.9)$ & 0.468 & 1.9 \\
\hline Hypertension & $1,544(50.1)$ & $1,552(50.4)$ & 0.838 & 0.5 \\
\hline Dyslipidemia & $253(8.2)$ & $259(8.4)$ & 0.782 & 0.7 \\
\hline Current or ex-smoking & $1,927(62.6)$ & $1,911(62.1)$ & 0.674 & 1.1 \\
\hline Chronic kidney disease & $472(15 \cdot 3)$ & $475(15 \cdot 4)$ & 0.916 & 0.3 \\
\hline \multicolumn{5}{|l|}{ Previous medical history } \\
\hline History of MI & $120(3.9)$ & $117(3.8)$ & 0.842 & 0.5 \\
\hline History of angina & $149(4.8)$ & $152(4.9)$ & 0.859 & 0.4 \\
\hline History of heart failure & $23(0.7)$ & $20(0.6)$ & 0.646 & 1.1 \\
\hline History of CVA & $136(4 \cdot 4)$ & $141(4 \cdot 6)$ & 0.759 & 0.8 \\
\hline \multicolumn{5}{|c|}{ Clinical characteristics at presentation and In-hospital } \\
\hline Killip class $\geq$ III on admission & $300(9.7)$ & $302(9.8)$ & 0.932 & 0.2 \\
\hline STEMI & $1,669(54.2)$ & $1,637(53.2)$ & 0.413 & 2.1 \\
\hline Left ventricular ejection fraction & $51.9 \pm 10.5$ & $52.1 \pm 10.4$ & 0.619 & 1.3 \\
\hline \multicolumn{5}{|l|}{ Medication at discharge } \\
\hline Aspirin & $3,077(99 \cdot 9)$ & $3,077(99 \cdot 9)$ & 1.000 & 0.0 \\
\hline Clopidogrel & $2,098(68.1)$ & $2,155(70.0)$ & 0.116 & 4.0 \\
\hline Prasugrel & $360(11.7)$ & $350(11.4)$ & 0.690 & 1.0 \\
\hline Ticagrelor & $608(19 \cdot 7)$ & $556(18.1)$ & 0.091 & $4 \cdot 3$ \\
\hline RAS blockade & $2,693(87.5)$ & $2,735(88.8)$ & 0.098 & $3 \cdot 7$ \\
\hline Statins & $2,971(96.5)$ & $2,969(96.4)$ & 0.890 & 0.4 \\
\hline \multicolumn{5}{|c|}{ Angiographic and procedural characteristics } \\
\hline Glycoprotein IIb/IIIa inhibitor & $454(14 \cdot 7)$ & $450(14.6)$ & 0.885 & 0.4 \\
\hline Intracoronary imaging device & $658(21.4)$ & $651(21.1)$ & 0.827 & 0.5 \\
\hline LM or LAD infarct-related artery & $1,542(50.1)$ & $1,543(50.1)$ & 0.980 & 0.1 \\
\hline ACC/AHA B2/C lesion & $2,700(87.7)$ & $2,677(86.9)$ & 0.378 & 2.3 \\
\hline Pre-procedural TIMI flow grade ০-1 & $1,782(57 \cdot 9)$ & $1,766(57 \cdot 4)$ & 0.680 & 1.1 \\
\hline Post-procedural TIMI flow grade 3 & $3,004(97 \cdot 6)$ & $3,006(97.6)$ & 0.868 & 0.4 \\
\hline Stent diameter, mm & $3.14 \pm 0.43$ & $3.14 \pm 0.44$ & 0.851 & 0.5 \\
\hline Total stent length, mm & $29 \cdot 5 \pm 14.2$ & $29.6 \pm 14.0$ & 0.912 & 0.3 \\
\hline Multi-vessel coronary artery disease & $1,572(51.1)$ & $1,565(50.8)$ & 0.858 & 0.5 \\
\hline Cardiogenic shock & $154(5.0)$ & $154(5.1)$ & 1.000 & 2.0 \\
\hline Mechanical circulatory support & $65(2.1)$ & $56(1.8)$ & 0.409 & 2.1 \\
\hline
\end{tabular}

Values are presented as mean \pm SD or number (\%).

MI, myocardial infarction; CVA, cerebrovascular accident; STEMI, ST segment elevation myocardial infarction; RAS, renin-angiotensin system; LM, left main; LAD, left anterior descending; ACC/AHA, American College of Cardiology/American Heart Association; TIMI, thrombolysis in myocardial infarction. 
Table 2. Clinical outcomes of propensity score-matched patients according to treatment at discharge and during follow-up

\begin{tabular}{|c|c|c|c|c|c|c|}
\hline & $\begin{array}{l}\text { Vasodilating } \\
\beta \text {-blockers } \\
(\mathrm{n}=3,079)\end{array}$ & $\begin{array}{c}\text { Conventional } \\
\beta \text {-blockers } \\
(\mathrm{n}=3,079)\end{array}$ & $\begin{array}{l}\text { Unadjusted } \\
\mathrm{HR}(95 \% \mathrm{CI})\end{array}$ & $p$ value & $\begin{array}{c}\text { Adjusted }^{\mathrm{a}} \\
\operatorname{HR}(95 \% \mathrm{CI})\end{array}$ & $p$ value \\
\hline $\begin{array}{l}\text { Cardiac death, myocardial } \\
\text { infarction, or hospitalisation } \\
\text { for heart failure }\end{array}$ & $235(7.6)$ & $302(9.8)$ & $0.77(0.65-0.91)$ & 0.003 & $0.76(0.64-0.90)$ & 0.002 \\
\hline Cardiac death & $108(3.5)$ & $147(4.8)$ & $0.73(0.57-0.94)$ & 0.015 & $0.73(0.57-0.94)$ & 0.013 \\
\hline Myocardial infarction & $75(2.4)$ & $93(3.0)$ & $0.80(0.59-1.09)$ & 0.160 & $0.80(0.59-1.09)$ & 0.155 \\
\hline $\begin{array}{l}\text { Hospitalisation for heart } \\
\text { failure }\end{array}$ & $81(2.6)$ & $98(3.2)$ & $0.82(0.61-1.10)$ & 0.192 & $0.81(0.61-1.09)$ & 0.170 \\
\hline
\end{tabular}

Values are presented as number (\%).

$\mathrm{HR}$, hazard ratio; CI, confidence interval.

aAdjusted for age, sex, hypertension, diabetes mellitus, dyslipidemia, current or ex-smoking, chronic kidney disease, left main or left anterior descending as infarct related artery, multivessel disease, left ventricular ejection fraction, ST elevation myocardial infarction or non-ST elevation myocardial infarction, and use of renin angiotensin system blocker, statin.

\section{Subgroup analysis}

We calculated the HR for primary outcome in various subgroups in both propensity score-matched and whole population. No significant interactions between the use of vasodilating $\beta$-blockers and primary outcome were observed in most subgroups except the mode of presentation (Fig. 3 and Supplementary Fig. 2). Interestingly, the efficacy of vasodilating $\beta$-blockers was significantly prominent in STEMI patients than in NSTEMI patients. Of note, the benefit of vasodilating $\beta$-blockers was not affected by the presence of left ventricular dysfunction.

\section{DISCUSSION}

In this observational study using a nation-wide Korean multicenter registry, treatment with vasodilating $\beta$-blocker was found to be associated with better 3-year clinical outcomes in AMI patients who had underwent PCI. In our previous study, vasodilating $\beta$-blocker therapy was associated with significantly better 1-year clinical outcomes than those with conventional $\beta$-blocker therapy [13]. The present analysis confirmed the long-term benefits of vasodilating $\beta$-blockers.

In the Losartan Intervention For Endpoint (LIFE) trial with 9,193 hypertensive patients, atenolol, a conventional $\beta$-blocker showed worse outcome of the primary composite endpoint when compared with losartan, de- spite the similar reduction in blood pressure during a median follow-up of 4.8 years. Although this difference was mainly driven by stroke, without significant differences in the incidence rates of cardiovascular death and MI, atenolol was associated with a significantly higher rate of new-onset diabetes mellitus [15]. In the Clopidogrel and Metoprolol in Myocardial Infarction Trial (COMMIT) study, the effect of early intravenous metoprolol therapy was compared with that of placebo in 45,582 patients with STEMI; no difference in mortality was found (odds ratio [OR], o.99; 95\% CI, 0.92 to 1.05; $p=$ $0.69)$ during mean follow-up of 15 days. Metoprolol therapy resulted in less incidence of re-infarction (OR, o.82; $95 \% \mathrm{CI}, 0.72$ to $0.92 ; p=0.001)$ and ventricular fibrillation (OR, 0.83 ; 95\% CI, 0.75 to $0.93 ; p=0.001$ ), but the benefit was counterbalanced by higher incidence of cardiogenic shock (OR, 1.30; 95\% CI, 1.19 to 1.41; $p<0.001$ ) [16]. In the Anglo-Scandinavian Cardiac Outcomes Trial-Blood Pressure Lowering Arm (ASCOT-BPLA) trial with 19,257 hypertensive patients, the atenolol-based regimen led to a significant increase in the incidence of major cardiovascular events and diabetes mellitus compared with the amlodipine-based regimen [17]. All of these three major studies with conventional $\beta$-blockers failed to show the benefits of $\beta$-blockers over their competitors.

By contrast, in the Carvedilol Post Infarction Survival Control in Left Ventricular Dysfunction (CAPRICORN) trial conducted in 1,959 patients with AMI and LVEF 

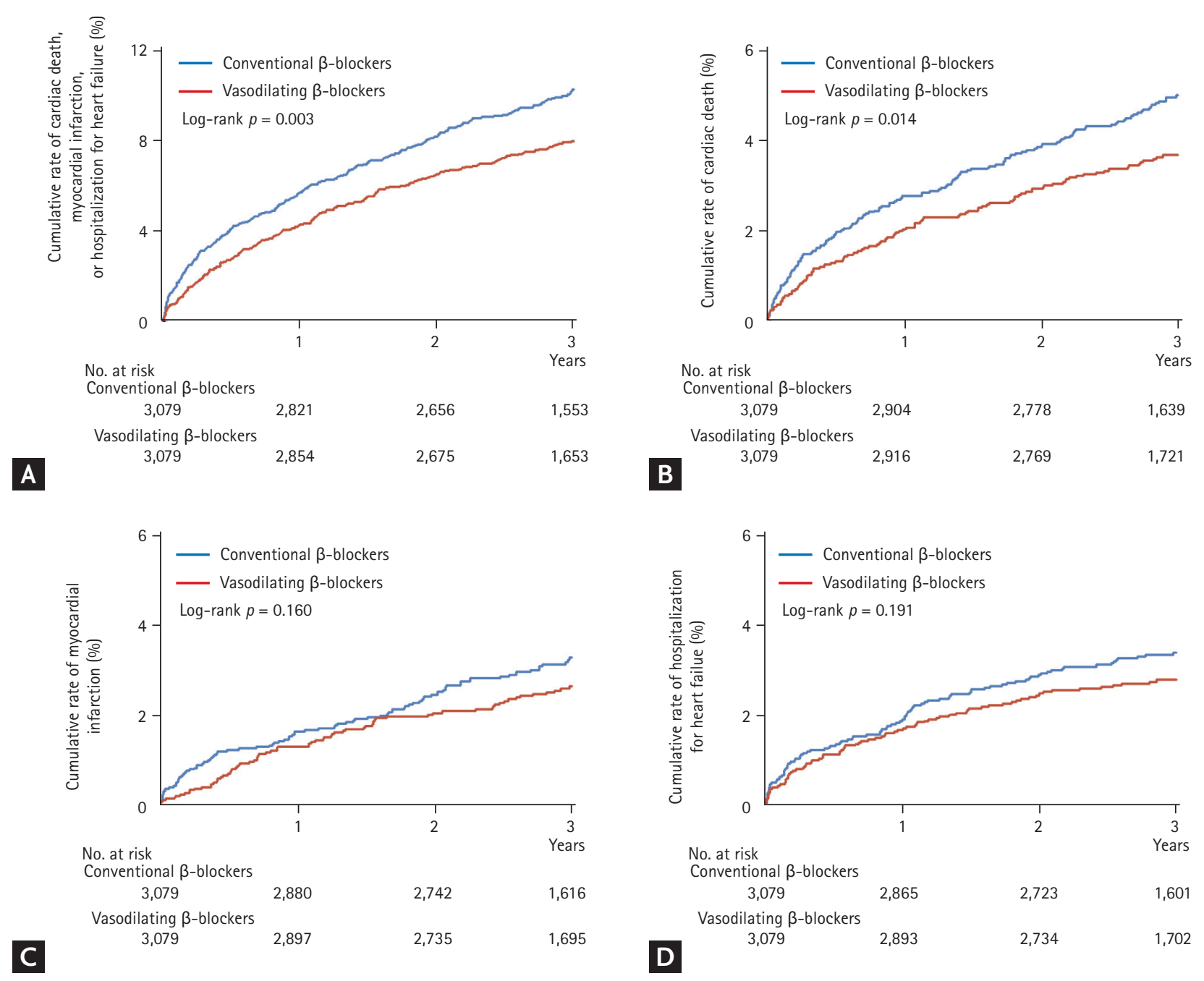

Figure 2. Kaplan-Meier curves for 3-year clinical outcomes in vasodilating versus conventional $\beta$-blocker groups in propensity score-matched patients. (A) A composite of cardiac death, myocardial infarction, or hospitalization for heart failure. (B) Cardiac death. (C) Myocardial infarction. (D) Hospitalization for heart failure.

$<40 \%$, carvedilol therapy decreased all-cause mortality (HR, 0.77; 95\% CI, 0.60 to 0.98; $p=0.03$ ) and incidence of non-fatal MI during a mean follow-up of 1.3 years compared with placebo [18]. The Carvedilol Or Metoprolol European Trial (COMET) performed in 1,511 chronic HF patients demonstrated that carvedilol therapy significantly reduced the all-cause mortality compared with metoprolol therapy [19]. In the Multicenter Automatic Defibrillator Implantation Trial With Cardiac Resynchronization Therapy (MADIT-CRT) trial performed in HF patients with devices, carvedilol therapy was associated with a $30 \%$ reduction in hospitalization for $\mathrm{HF}$ or death compared with metoprolol therapy [20]. Addi- tionally, this risk reduction was more pronounced in the subgroup of cardiac resynchronization therapy with implantable cardioverter-defibrillator (CRT-D) patients and CRT-D patients with left bundle branch block [20]. Unlike previous studies with conventional $\beta$-blockers, which failed to demonstrate the benefits of $\beta$-blocker therapy, the studies with newer vasodilating $\beta$-blocker therapy showed better clinical outcomes.

Possible explanation for these results may come from many beneficial effects of vasodilating $\beta$-blockers, including the effects on central blood pressure and metabolic derangement. Conventional $\beta$-blockers can increase central blood pressure, whereas vasodilating 


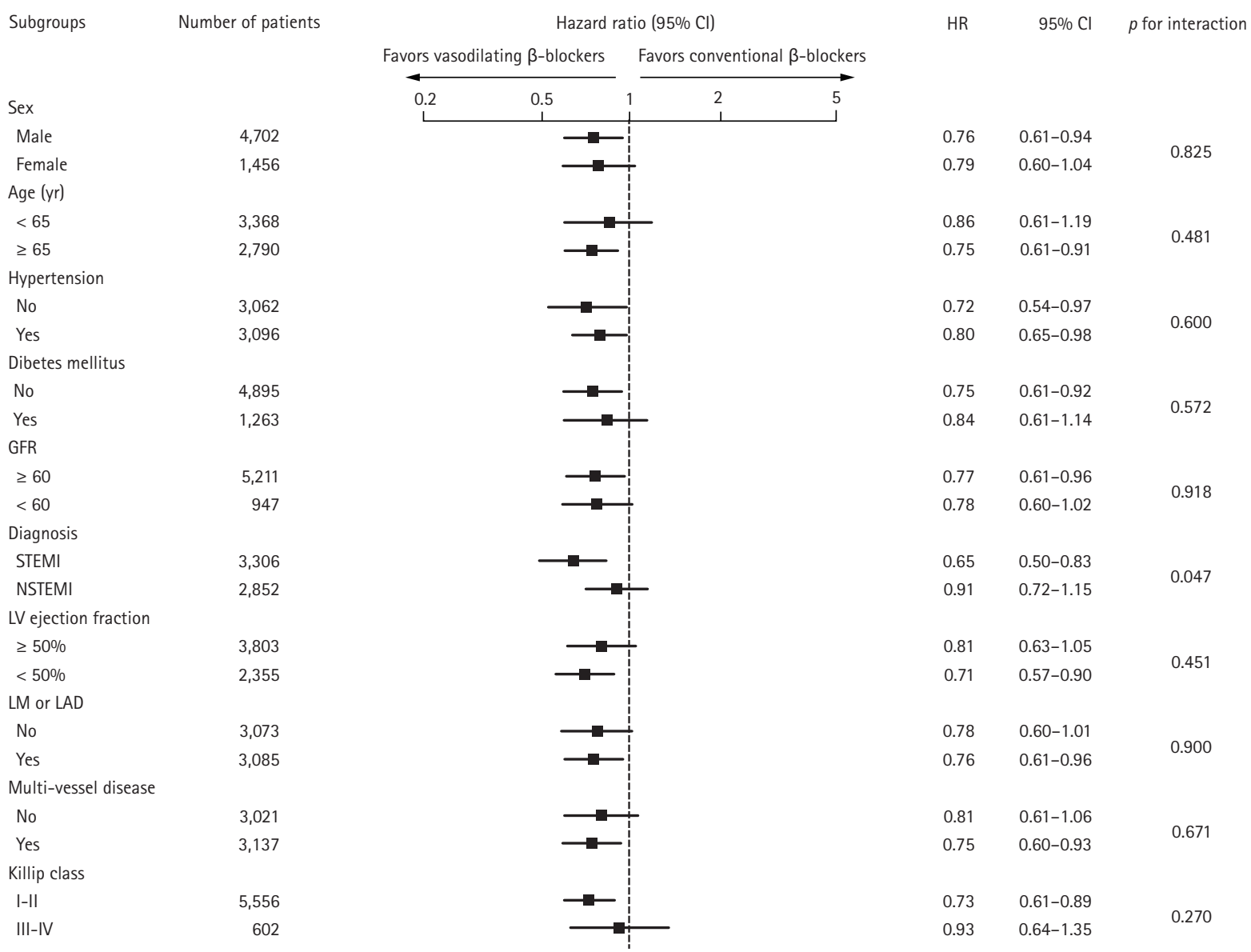

Figure 3. Comparative unadjusted hazard ratios (HRs) of composite cardiac events for subgroups in propensity score-matched patients using vasodilating $\beta$-blockers and conventional $\beta$-blockers. CI, confidence interval; GFR, glomerular filtration rate (mg/dL); STEMI, ST segment elevation myocardial infarction; NSTEMI, non-ST segment elevation myocardial infarction; LV, left ventricle; LM, left main; LAD, left anterior descending artery.

$\beta$-blockers can decrease central blood pressure [11]. In addition, vasodilating $\beta$-blockers do not have a negative effect on insulin sensitivity and lipid control in contrast to conventional $\beta$-blockers [21]. Furthermore, vasodilating $\beta$-blockers improve the endothelial function, arterial stiffness, anti-oxidant function, anti-inflammatory function, and inhibit platelet aggregation by increasing the bioactivity of endothelium-derived nitric oxide [12,22-24]. Vasodilating $\beta$-blockers can also improve coronary microvascular function, coronary flow reserve compared with conventional $\beta$-blockers [25,26]. Moreover, vasodilating $\beta$-blockers can improve exercise tolerance, time to onset of angina, and ST segment change during exercise stress test, compared with conventional $\beta$-blockers $[27,28]$. These various favorable mechanisms as a whole may explain why better outcomes of a composite ebndopoints were mainly dirven by cardiac death in our study, but are not solely affected by MI or HF.

We speculate that it takes time for the beneficial effects of vasodilating $\beta$-blockers to be translated into better clinical outcomes. Our current study showed the long-term benefits of vasodilating $\beta$-blockers on the clinical outcomes compared with conventional $\beta$-blockers over a 3-year follow-up.

Several limitations should be taken into account when these results are interpreted. First, our study has general limitations inherent to a non-randomized trial. Although we performed a propensity score matching and multivariable adjustment, other unmeasured variables could affect the results. Second, this registry did not al- 
low us to reliably assess the effects of $\beta$-blocker dosage. However, because this real-world prospective registry may reflect routine clinical practice, we surmise that the dose of $\beta$-blockers was optimally adjusted depending on the patients' condition. Third, as we focused on longterm effect of vasodilating $\beta$-blockers over conventional $\beta$-blockers, only patients who survived during the index hospitalization were included in the analysis. Therefore, the role of vasodilating $\beta$-blockers in the early phase of AMI was not assessed in this study. Fourth, most of the $\beta$-blockers used in this study were carvedilol and bisoprolol. In contrast, the usage of nebivolol and metoprolol is not so common in the Republic of Korea. Thus, the results of our study should be cautiously applied in other countries where the prescription pattern of $\beta$-blockers is different from that in the Republic of Korea.

In conclusion, vasodilating $\beta$-blocker therapy in patients with AMI who had undergone PCI demonstrated long-term benefits at 3 years after the index event compared with conventional $\beta$-blocker therapy. Large-scale, randomized controlled trials are warranted to confirm this finding. Further studies are also needed to find how long (vasodilating) $\beta$-blocker therapy should be indicated in patients with AMI who had undergone PCI.

\section{KEY MESSAGE}

1. In real world practice, vasodilating $\beta$-blocker therapy in acute myocardial infarction (AMI) patients was associated with significantly lower risk of composite cardiac event, including cardiac death, myocardial infarction, or hospitalisation for heart failure compared with conventional $\beta$-blocker therapy at a long term (3 years) follow-up.

2. The results were not affected by subgroups: of note, vasodilating $\beta$-blockers were associated with better clinical outcomes not only in patients with left ventricular dysfunction but also in those without left ventricular dysfunction

3. Vasodilating $\beta$-blockers would be preferentially recommended over conventional $\beta$-blockers for AMI patients.

\section{Conflict of interest}

No potential conflict of interest relevant to this article was reported.

\section{Acknowledgments}

This research was supported by a fund (2016-ER6304-02) for Research of Korea Centers for Disease Control and Prevention. Sponsors had no role in the study design or data analysis and interpretation or in the decision to submit the manuscript for publication.

\section{REFERENCES}

1. Amsterdam EA, Wenger NK, Brindis RG, et al. 2014 AHA/ ACC guideline for the management of patients with non-ST-elevation acute coronary syndromes: a report of the American College of Cardiology/American Heart Association Task Force on Practice Guidelines. Circulation 2014;130:e344-e426.

2. Ibanez B, James S, Agewall S, et al. 2017 ESC guidelines for the management of acute myocardial infarction in patients presenting with ST-segment elevation: the task force for the management of acute myocardial infarction in patients presenting with ST-segment elevation of the European Society of Cardiology (ESC). Eur Heart J 2018;39:119-177.

3. O'Gara PT, Kushner FG, Ascheim DD, et al. 2013 ACCF/ AHA guideline for the management of ST-elevation myocardial infarction: a report of the American College of Cardiology Foundation/American Heart Association Task Force on Practice Guidelines. Circulation 2013;127:e362-e425.

4. Roffi M, Patrono C, Collet JP, et al. 2015 ESC guidelines for the management of acute coronary syndromes in patients presenting without persistent ST-segment elevation: Task Force for the Management of Acute Coronary Syndromes in Patients Presenting without Persistent ST-Segment Elevation of the European Society of Cardiology (ESC). Eur Heart J 2016;37:267-315.

5. Giugliano D, Acampora R, Marfella R, et al. Metabolic and cardiovascular effects of carvedilol and atenolol in non-insulin-dependent diabetes mellitus and hypertension. A randomized, controlled trial. Ann Intern Med 1997;126:955-959.

6. Bakris GL, Fonseca V, Katholi RE, et al. Metabolic effects 
of carvedilol vs metoprolol in patients with type 2 diabetes mellitus and hypertension: a randomized controlled trial. JAMA 2004;292:2227-2736.

7. Poirier L, Cleroux J, Nadeau A, Lacourciere Y. Effects of nebivolol and atenolol on insulin sensitivity and haemodynamics in hypertensive patients. J Hypertens 2001;19:1429-1435.

8. Bank AJ, Kelly AS, Thelen AM, Kaiser DR, Gonzalez-Campoy JM. Effects of carvedilol versus metoprolol on endothelial function and oxidative stress in patients with type 2 diabetes mellitus. Am J Hypertens 2007;20:777-783.

9. Stauffer BL, Dow CA, Diehl KJ, Bammert TD, Greiner JJ, DeSouza CA. Nebivolol, but not metoprolol, treatment improves endothelial fibrinolytic capacity in adults with elevated blood pressure. J Am Heart Assoc 2017;6:e007437.

10. Feuerstein GZ, Ruffolo RR Jr. Carvedilol, a novel multiple action antihypertensive agent with antioxidant activity and the potential for myocardial and vascular protection. Eur Heart J 1995;16 Suppl F:38-42.

11. McEniery CM, Cockcroft JR, Roman MJ, Franklin SS, Wilkinson IB. Central blood pressure: current evidence and clinical importance. Eur Heart J 2014;35:1719-1725.

12. Munzel T, Gori T. Nebivolol: the somewhat-different beta-adrenergic receptor blocker. J Am Coll Cardiol 2009;54:1491-1499.

13. Chung J, Han JK, Kim YJ, et al. Benefit of vasodilating $\beta$-blockers in patients with acute myocardial infarction after percutaneous coronary intervention: nationwide multicenter cohort study. J Am Heart Assoc 2017;6:0007063.

14. Chen KY, Rha SW, Li YJ, et al. Triple versus dual antiplatelet therapy in patients with acute ST-segment elevation myocardial infarction undergoing primary percutaneous coronary intervention. Circulation 2009;119:3207-3214.

15. Dahlof B, Devereux RB, Kjeldsen SE, et al. Cardiovascular morbidity and mortality in the Losartan Intervention For Endpoint reduction in hypertension study (LIFE): a randomised trial against atenolol. Lancet 2002;359:995-1003.

16. Chen ZM, Pan HC, Chen YP, et al. Early intravenous then oral metoprolol in 45,852 patients with acute myocardial infarction: randomised placebo-controlled trial. Lancet 2005;366:1622-1632.

17. Dahlof B, Sever PS, Poulter NR, et al. Prevention of cardiovascular events with an antihypertensive regimen of amlodipine adding perindopril as required versus aten- olol adding bendroflumethiazide as required, in the Anglo-Scandinavian Cardiac Outcomes Trial-Blood Pressure Lowering Arm (ASCOT-BPLA): a multicenter randomised controlled trial. Lancet 2005;366:895-906.

18. Dargie HJ. Effect of carvedilol on outcome after myocardial infarction in patients with left-ventricular dysfunction: the CAPRICORN randomised trial. Lancet 2001;357:138513-138590.

19. Poole-Wilson PA, Swedberg K, Cleland JG, et al. Comparison of carvedilol and metoprolol on clinical outcomes in patients with chronic heart failure in the Carvedilol Or Metoprolol European Trial (COMET): randomised controlled trial. Lancet 2003;362:7-13.

20. Ruwald MH, Ruwald AC, Jons C, et al. Effect of metoprolol versus carvedilol on outcomes in MADIT-CRT (multicenter automatic defibrillator implantation trial with cardiac resynchronization therapy). J Am Coll Cardiol 2013;61:1518-1526.

21. Fonseca VA. Effects of beta-blockers on glucose and lipid metabolism. Curr Med Res Opin 2010;26:615-629.

22. Giugliano D, Marfella R, Acampora R, Giunta R, Coppola L, D'Onofrio F. Effects of perindopril and carvedilol on endothelium-dependent vascular functions in patients with diabetes and hypertension. Diabetes Care 1998;21:631-636.

23. Falciani M, Rinaldi B, D'Agostino B, et al. Effects of nebivolol on human platelet aggregation. J Cardiovasc Pharmacol 2001;38:922-929.

24. Lopez BL, Christopher TA, Yue TL, Ruffolo R, Feuerstein GZ, Ma XL. Carvedilol, a new beta-adrenoreceptor blocker antihypertensive drug, protects against free-radical-induced endothelial dysfunction. Pharmacology 1995:51:165173 .

25. Xiaozhen H, Yun Z, Mei Z, Yu S. Effect of carvedilol on coronary flow reserve in patients with hypertensive left-ventricular hypertrophy. Blood Press 2010;19:40-47.

26. Gullu H, Erdogan D, Caliskan M, et al. Different effects of atenolol and nebivolol on coronary flow reserve. Heart 2006;92:1690-1691.

27. van der Does R, Hauf-Zachariou U, Pfarr E, et al. Comparison of safety and efficacy of carvedilol and metoprolol in stable angina pectoris. Am J Cardiol 1999;83:643-649.

28. Van Bortel LM, van Baak MA. Exercise tolerance with nebivolol and atenolol. Cardiovasc Drugs Ther 1992;6:239247. 
Supplementary Table 1. Baseline characteristics of study patients according to treatment at discharge and during follow-up

\begin{tabular}{|c|c|c|c|c|}
\hline Variable & $\begin{array}{l}\text { Vasodilating } \beta \text {-blockers } \\
(\mathrm{n}=3,608)\end{array}$ & $\begin{array}{c}\text { Conventional } \\
\beta \text {-blockers }(n=3,661)\end{array}$ & $p$ value & $\begin{array}{c}\text { Standardized } \\
\text { difference }\end{array}$ \\
\hline \multicolumn{5}{|l|}{ Demographics } \\
\hline Age, yr & $62.3 \pm 12.4$ & $63.2 \pm 12.4$ & 0.002 & 7.8 \\
\hline Male sex & $2,779(77.0)$ & $2,740(74.8)$ & 0.030 & 8.6 \\
\hline \multicolumn{5}{|l|}{ Coronary risk factors } \\
\hline Diabetes mellitus & $689(19.1)$ & $855(23.4)$ & $<0.001$ & 11.3 \\
\hline Hypertension & $1,804(50.0)$ & $1,909(52.1)$ & 0.067 & 6.0 \\
\hline Dyslipidemia & $291(8.1)$ & $305(8.3)$ & 0.680 & 0.4 \\
\hline Current or ex-smoking & $2,212(62.8)$ & $2,176(60.7)$ & 0.012 & 6.0 \\
\hline Chronic kidney disease & $546(15.1)$ & $634(17 \cdot 3)$ & 0.011 & $7 \cdot 3$ \\
\hline \multicolumn{5}{|l|}{ Previous medical history } \\
\hline History of MI & $140(3.9)$ & $148(4.0)$ & 0.723 & 0.8 \\
\hline History of Angina & $184(5.1)$ & $181(4 \cdot 9)$ & 0.761 & 0.1 \\
\hline History of heart failure & $30(0.8)$ & $22(0.6)$ & 0.241 & 1.6 \\
\hline History of CVA & $153(4 \cdot 2)$ & $176(4.8)$ & 0.240 & 3.8 \\
\hline \multicolumn{5}{|c|}{ Clinical characteristics at presentation and in-hospital } \\
\hline Killip class $\geq$ III on admission & $329(9.1)$ & $437(11.9)$ & $<0.001$ & 9.8 \\
\hline STEMI & $2,004(55 \cdot 5)$ & $1,859(50.8)$ & $<0.001$ & $9 \cdot 4$ \\
\hline Left ventricular ejection fraction & $51.5 \pm 10.5$ & $52.5 \pm 10.5$ & $<0.001$ & $9 \cdot 7$ \\
\hline \multicolumn{5}{|l|}{ Medication at discharge } \\
\hline Aspirin & $3,603(99 \cdot 9)$ & $3,659(99.9)$ & 0.249 & 1.6 \\
\hline Clopidogrel & $2,470(68.5)$ & $2,548(69.6)$ & 0.293 & 2.5 \\
\hline Prasugrel & $331(9.2)$ & $474(12.9)$ & $<0.001$ & 12.1 \\
\hline Ticagrelor & $781(21.6)$ & $626(17.1)$ & $<0.001$ & 11.5 \\
\hline RAS blockade & $3,020(83.7)$ & $3,285(89.7)$ & $<0.001$ & 16.7 \\
\hline Statins & $3,485(96.6)$ & $3,477(95.0)$ & 0.001 & 9.1 \\
\hline \multicolumn{5}{|c|}{ Angiographic and procedural characteristics } \\
\hline Glycoprotein IIb/IIIa inhibitor & $527(14.6)$ & $535(14.6)$ & 0.993 & 0.7 \\
\hline Intracoronary imaging device & $799(22.1)$ & $750(20.5)$ & 0.084 & 5.2 \\
\hline LM or LAD infarct-related artery & $1,831(50.7)$ & $1,814(49 \cdot 5)$ & 0.307 & 2.1 \\
\hline ACC/AHA B2/C lesion & $3,612(87 \cdot 6)$ & $3,171(86.6)$ & 0.193 & 2.4 \\
\hline Pre-procedural TIMI flow grade o-1 & $2,083(57 \cdot 7)$ & $2,093(57.2)$ & 0.628 & 2.4 \\
\hline Post-procedural TIMI flow grade 3 & $3,516(97 \cdot 5)$ & $3,577(97 \cdot 7)$ & 0.479 & 2.5 \\
\hline Stent diameter, mm & $3.13 \pm 0.43$ & $3.15 \pm 0.44$ & 0.165 & 3.6 \\
\hline Total stent length, mm & $29.4 \pm 13.9$ & $30.0 \pm 14.6$ & 0.086 & $3 \cdot 5$ \\
\hline Multi-vessel coronary artery disease & $1,900(52.7)$ & $1,831(50.0)$ & 0.024 & $5 \cdot 9$ \\
\hline Cardiogenic shock & $173(4.8)$ & $226(6.2)$ & 0.010 & 6.1 \\
\hline Mechanical circulatory support & $82(2.3)$ & $71(1.9)$ & 0.322 & 1.1 \\
\hline
\end{tabular}

Values are presented as mean $\pm \mathrm{SD}$ or number (\%).

MI, myocardial infarction; CVA, cerebrovascular accident; STEMI, ST segment elevation myocardial infarction; RAS, renin-angiotensin system; LM, left main; LAD, left anterior descending; ACC/AHA, American College of Cardiology/American Heart Association; TIMI, thrombolysis in myocardial infarction. 
Chung J, et al. Benefit of vasodilating beta-blocker

Supplementary Table 2. Clinical outcomes of study patients according to treatment at discharge and during follow-up

\begin{tabular}{|c|c|c|c|c|c|c|}
\hline & $\begin{array}{c}\text { Vasodilating } \\
\beta \text {-blockers } \\
(\mathrm{n}=3,608)\end{array}$ & $\begin{array}{c}\text { Conventional } \\
\beta \text {-blockers } \\
(\mathrm{n}=3,661)\end{array}$ & $\begin{array}{c}\text { Unadjusted } \\
\text { HR } \\
(95 \% \mathrm{CI})\end{array}$ & $p$ value & $\begin{array}{c}\text { Adjusted }^{\mathrm{a}} \\
\text { HR } \\
(95 \% \mathrm{CI})\end{array}$ & $p$ value \\
\hline $\begin{array}{l}\text { Cardiac death, myocardial infarction, } \\
\text { or hospitalisation for heart failure }\end{array}$ & $282(7.8)$ & $359(9.8)$ & $0.79(0.68-0.92)$ & 0.003 & $0.80(0.68-0.94)$ & 0.006 \\
\hline Cardiac death & $128(3 \cdot 5)$ & $176(4.8)$ & $0.74(0.59-0.92)$ & 0.008 & $0.78(0.61-0.99)$ & 0.038 \\
\hline Myocardial infarction & $90(2.5)$ & $112(3.1)$ & $0.81(0.61-1.07)$ & 0.140 & $0.82(0.62-1.09)$ & 0.174 \\
\hline Hospitalisation for heart failure & $100(2.8)$ & $115(3.1)$ & $0.88(0.67-1.15)$ & 0.336 & $0.86(0.65-1.13)$ & 0.275 \\
\hline
\end{tabular}

HR, hazard ratio; CI, confidence interval.

${ }^{a}$ Adjusted for age, sex, hypertension, diabetes mellitus, dyslipidemia, current or ex-smoking, chronic kidney disease, left main or left anterior descending as infarct related artery, multivessel disease, left ventricular ejection fraction, ST elevation myocardial infarction or non-ST elevation myocardial infarction, and use of renin angiotensin system blocker, statin. 

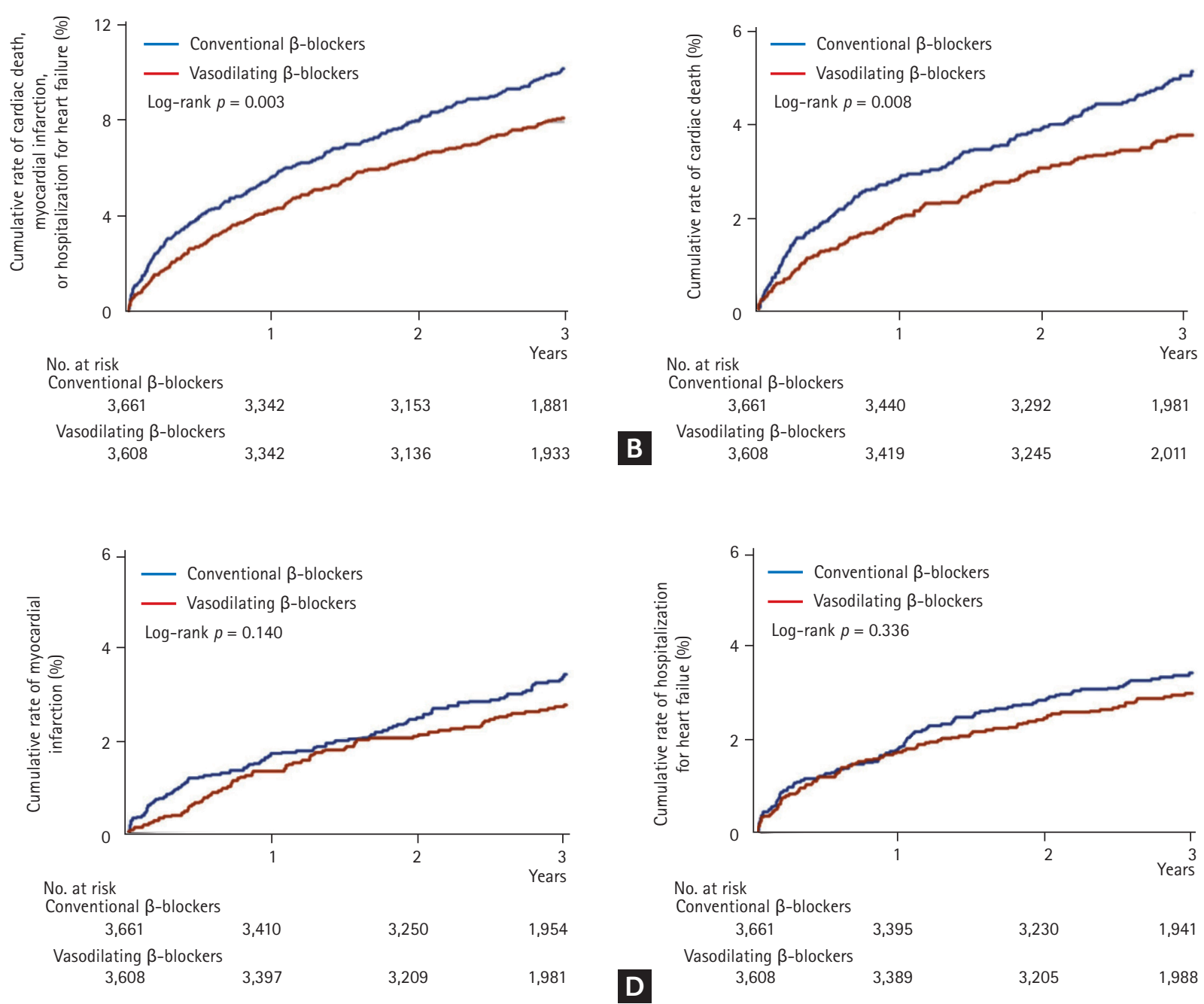

Supplementary Figure 1. Kaplan-Meier curves for 3-year clinical outcomes in vasodilating versus conventional $\beta$-blocker groups in the whole study population. (A) A composite of cardiac death, myocardial infarction, or hospitalization for heart failure. (B) Cardiac death. (C) Myocardial infarction. (D) Hospitalization for heart failure. 


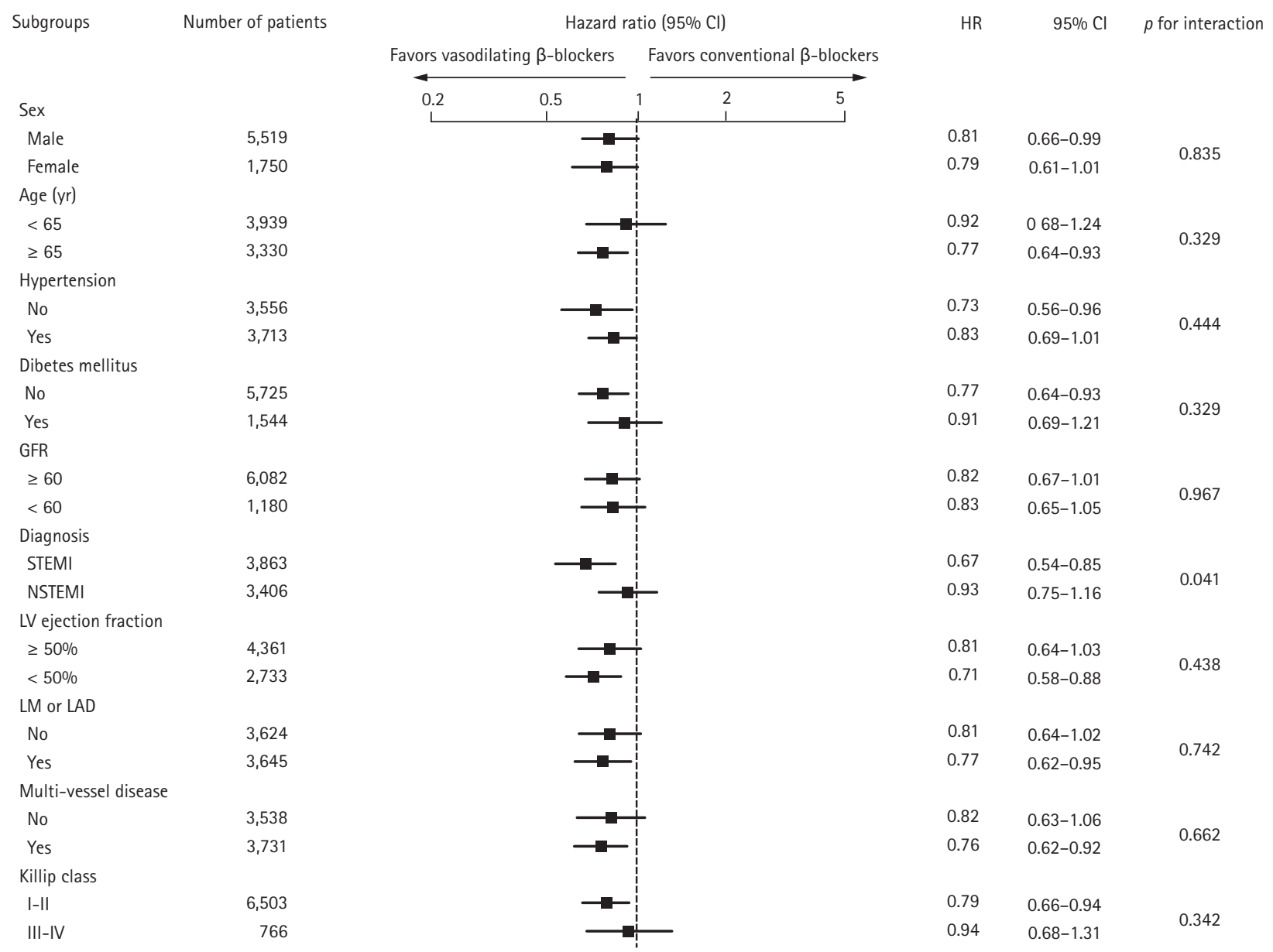

Supplementary Figure 2. Comparative unadjusted hazard ratios (HRs) of composite cardiac events for subgroups in the whole study population using vasodilating $\beta$-blockers and conventional $\beta$-blockers. CI, confidence interval; GFR, glomerular filtration rate (mg/dL); STEMI, ST segment elevation myocardial infarction; NSTEMI, non-ST segment elevation myocardial infarction; LV, left ventricle; LM, left main; LAD, left anterior descending artery. 